\title{
Reactivation of Tuberculosis in Three Cases of Psoriasis after Initiation of Anti-TNF Therapy
}

\author{
Samya Abu Shaikha Khalid Mansour Hassan Riad \\ Department of Dermatology, Hamad Medical Corporation, Doha, Qatar
}

Key Words

Psoriasis · Tuberculosis · Biologics · Reactivation

\begin{abstract}
New biological therapies for disabling diseases such as psoriasis may carry both short- and long-term risks. Tuberculosis (TB) reactivation is a frequent complication of anti-tumor necrosis factor (TNF) therapy. We present 3 cases of psoriasis that were treated with different types of anti-TNF and developed TB infection (TBI) during therapy. One of the cases was diagnosed as active pulmonary TB and the other 2 cases as latent TBI. All cases received appropriate anti-TB treatment, and the anti-TNF therapy was interrupted and then resumed according to various clinical considerations.
\end{abstract}

\section{Introduction}

Tuberculosis (TB) infections (TBI) affect almost one third of the world population. In 2010, there were about 8.8 million (range 8.5-9.2) incident cases of TB [1]. The incidence of TB in Qatar during the last decade remained almost unchanged (43-47 cases per 100,000 per year) [2], despite two major changes: a threefold increase in the population size and the introduction of new immunosuppressing agents, mainly antitumor necrosis factors (TNFs), by various sub-specialties.

The lifetime incidence of TB in untreated psoriatic patients is unknown. Studies on risk factors causing TB did not show that psoriatic patients are at greater risk of acquiring TB compared to the general population; however, psoriatic patients are more likely to be treated for latent TBI (LTBI) before anti-TNF therapy compared to patients with inflammatory bowel disease [3]. Moreover, in one study the in vitro T cell responsiveness was diminished towards Mycobacterium tuberculosis in cases with severe psoriasis [4]. 
TNF is crucial for immunity against TBI, especially in granuloma formation and in maintaining the integrity of granuloma. Before initiation of anti-TNF therapy in psoriatic patients, screening for LTBI using the tuberculin skin test (TST), interferon gamma release assay (IGRA) and chest X-ray (CXR) revealed a positive reaction in about $20 \%$ of the cases in the 2009 Swiss study by Laffitte et al. [5]. In a study from Greece using TST alone, $50 \%$ of the patients showed a positive tuberculin test [3]. In a wide-scale study from Italy using IGRA and involving 405 psoriatic patients, $18 \%$ of the samples showed a positive reaction [6].

Complications of biologics are theoretically few, but practically, and with the increasing use of those agents, they are no longer rare. Some complications may also be fatal or life threatening. Post-marketing surveillance and case reports contribute to shedding light on problems that occur in real life, as opposed to in well-designed studies done on selected cases or volunteers. Pooling the safety data from studies on biologics from different conditions is worthwhile.

Although screening is effective in the diagnosis of most cases of LTBI prior to antiTNF therapy, the risk of developing active TB or LTBI after initiation of therapy is still to be considered. All anti-TNF agents have warning labels about the risk of infections, namely TB. We present here 3 cases that were sufficiently screened for TBI before initiation of anti-TNF treatment and later developed laboratory or clinical signs of infection.

\section{Case Report}

Case 1

A 47-year-old local male presented with an 8-year history of severe disabling palmoplantar psoriasis. He was both hypertensive and diabetic (controlled adult onset). He had no previous history of systemic medications and showed low compliance with phototherapy. He was mainly treated with local applications of steroids, tar, salicylic acid and calcipotriol. The patient was started on acitretin $50 \mathrm{mg}$ daily and $50 \%$ urea cream with only marginal response after 18 weeks. Due to the severity of the condition, the patient was screened before etanercept was added. All tests were within normal range, including negative TST and CXR. The patient completed 12 weeks with biweekly $50-\mathrm{mg}$ subcutaneous injections, with minor improvement, followed by 12 weeks on once weekly $50 \mathrm{mg}$ etanercept without significant change. Both drugs were discontinued and the patient was screened again before switching to another anti-TNF; this time IGRA was performed and the result was negative. He started adalimumab therapy and showed a good and stable response for 14 weeks until he developed fever, night sweating, chills, loss of appetite and persistent cough. The condition evolved rapidly, necessitating hospitalization. Bronchial lavage and pleural effusion tabbing of the right lung were both positive for TB by polymerase chain reaction. Anti-TB therapy was started and adalimumab was discontinued. Six weeks later, the chest condition showed marked improvement, while palmoplantar psoriasis worsened and the patient's ability to walk was limited. Based on the recommendation of the pulmonologist and the patient's consent, adalimumab was resumed on the condition of strict compliance with anti-TB therapy and close follow-up by the medical staff. The patient was followed up for 26 weeks, with complete clearance of both his chest condition and palmoplantar psoriasis. Follow-up of the patient will continue in order to rule out recurrence of TB.

Case 2

A 38-year-old local male presented with a 23-year history of generalized plaque psoriasis. His BMI was 42 , with a physician global assessment (PGA) of 4 . He suffered from joint pain in both small and large joints, with no radiological findings. He had a positive family history but no history of systemic medications. The patient was screened before administration of infliximab therapy. All tests were within normal range, including IGRA and CXR. Infliximab was initiated with good response and a cessation of joint pain. No side effects were noted in the first year of therapy. During his annual 
checkup, an IGRA showed a positive conversion though CXR and microbiology for TB were negative; the patient was asymptomatic. LTBI was diagnosed by the pulmonologist and anti-TB treatment was started, with a 1-month cessation of infliximab therapy. The patient was followed up for another year; his condition is stable, with a continued response to infliximab and no side effects. Follow-up of the patient will continue in order to rule out recurrence of TB.

Case 3

A 33-year-old local male presented with generalized psoriasis of PGA 3. He is the younger brother of case 2, however, with a less severe condition, lower BMI and no associated comorbidities. He was treated with short, irregular courses of narrow-band UVB due to poor compliance and no history of systemic therapy. The patient was screened before administration of etanercept injection. All tests were within normal limits, including IGRA and CXR. The patient showed good response to therapy; during his annual checkup he also showed a positive conversion of IGRA, while CXR and microbiology were negative for TB. He was referred to a pulmonologist who diagnosed the case similarly as LTBI. Anti-TB therapy was given, with a 1-month cessation of etanercept. The patient was followed up for 8 months, with continued efficacy of resumed etanercept injections and no report of side effects. Follow-up of the patient will continue in order to rule out recurrence of TB (table 1).

\section{Discussion}

The complications of immunosuppression of biologics, particularly the anti-TNFs, can be placed into 3 main categories: infections, malignancies and miscellaneous. Infections are more frequent and evolve rapidly in short time spans compared to malignancies. Since TBI is highly prevalent worldwide, special care is required for the screening and monitoring for LTBI in patients on anti-TNF therapy. Most of the current guidelines recommend TST as the main screening tool for LTBI in psoriasis patients before anti-TNF- $\alpha$ therapy; however, the European S3-guidelines are more flexible and suggest the use of either TST or IGRA or both [7, 8]. There are few post-marketing reports on the development of both active TB and LTBI after initiation of anti-TNF therapy in psoriatic patients [9-11]. Patients may develop serious, life-threatening, active TBI quite rapidly and without clinical or investigative predictors.

Screening for LTBI before treatment with anti-TNF agents in psoriasis patients showed varying results $[3,5,6]$. The frequency of LTBI in patients with psoriasis after initiation of anti-TNF therapy is also variable across different studies [9-11]. In a Spanish study of 144 patients on the 3 anti-TNF agents, 29\% showed TST and reTST conversion after different periods of therapy [9]. In a study from Taiwan done on 147 patients using etanercept, adalimumab or both, $11 \%$ showed conversion of IGRA after a median period of 24 weeks of exposure [10]. Also, in an Italian study done on 50 patients using the 3 anti-TNFs as monotherapy or combined with methotrexate, 7 (14\%) showed IGRA conversion after 1 year [11]. In this study, 2 cases of IGRA conversion were discovered during an annual checkup of anti-TNF therapy maintenance and 1 case was diagnosed as active pulmonary TB 10 months after starting biologics.

All types of anti-TNF agents caused TB reactivation in these studies, regardless of a higher likelihood of causing LTBI or the duration of therapy. Our study is also in agreement with these studies, as it showed that the 3 approved anti-TNFs indicated for the therapy of psoriasis, namely infliximab, adalimumab and etanercept, can all reactivate TB in psoriatic patients in the relatively short period of time of less than or equal to 1 year. 
The switching of anti-TNFs has thus far not been proved to increase the risk to patients to acquire TB, but we have to emphasize that case 1 in this study switched from etanercept to adalimumab and we could not ascertain if adalimumab alone was responsible for the reactivation of $\mathrm{TB}$, or the prolonged cumulative effect of both drugs was the reason.

In this study, the patients did not receive any other immunosuppressants such as methotrexate or cyclosporine before or during therapy. They were all BCG vaccinated in their infancy. Except for the first case who was diabetic and the third case who had short, interrupted phototherapy sessions, the patients had no other causes of immunosuppression or risk factors for TBI. We can conclude that anti-TNF therapy was the main risk factor in developing TBI.

Due to possible false interpretation of the TST, especially in cases of immunosuppression, BCG-vaccinated patients and patients who have already started anti-TNF therapy, IGRA may have an advantage over TST in both sensitivity and specificity $[11,12]$. We retested our patients using IGRA and we suggest that, according to this study, it is better to retest patients after the first 6 months, and later annually.

One point of argument is that we were obliged to resume adalimumab in case 1 only 6 weeks after the start of a full course of multiple-drug therapy for TB. One of the comprehensive guidelines [13] stated that it is still debatable whether the anti-TNF agent can be restarted before receiving a full course of anti-TB treatment. Thus, whether to restart the anti-TNF agent within the time period ranging from a minimum of 6 weeks, the time course for a positive smear to turn negative, up to the standard of 9 months might be mainly a clinical decision. Re-initiation is case dependent and has many clinical considerations, such as the extent of TB disease and whether there are complications of TB that need special medical or surgical intervention. Other factors to be considered are that patient compliance and adherence to therapy is mandatory, as is acquiring a non-drug-resistant strain by culture and, lastly, having TB smears which have turned negative. However, ideally, a full 9 months of TB therapy is recommended before restarting anti-TNF therapy again.

One last point we should raise here is that rare cases which were initially diagnosed as LTBI, and having already received anti-TB therapy before the start of anti-TNF treatment, still developed active lymph node TB later [9]. Nevertheless, patients diagnosed and treated with active TB may develop recurrence due to drug resistance, which adds more responsibility and attention in monitoring these cases. Follow-up of our patients will continue in order to rule out the possibility of TB recurrence. 
Table 1. Clinical and laboratory data of the 3 cases

\begin{tabular}{|c|c|c|c|}
\hline & Case 1 & Case 2 & Case 3 \\
\hline Age, years & 47 & 38 & 33 \\
\hline Sex & male & male & male \\
\hline BMI & 30 & 42 & 36 \\
\hline Duration of psoriasis, years & 8 & 23 & 15 \\
\hline Special areas & palms and soles & scalp and flexures & scalp \\
\hline Psoriatic arthritis (joint) & no & yes & no \\
\hline Psoriasis PGA & 3 & 4 & 3 \\
\hline Comorbidity & diabetes and hypertension & massive obesity & obesity \\
\hline Family history & negative & positive & positive \\
\hline BCG vaccination & positive & positive & positive \\
\hline HIV status & negative & negative & negative \\
\hline \multicolumn{4}{|l|}{ Initial tests for TB } \\
\hline TST & negative & not done & not done \\
\hline IGRA & negative & negative & negative \\
\hline CXR & negative & negative & negative \\
\hline \multicolumn{4}{|l|}{ Post-therapy tests for TB } \\
\hline IGRA & positive & positive & positive \\
\hline CXR & positive & negative & negative \\
\hline Microbiology for TB & positive & negative & negative \\
\hline Diagnosis of TB status & active TB & LTBI & LTBI \\
\hline $\begin{array}{l}\text { Anti-TNF therapy } \\
\text { name and duration }\end{array}$ & $\begin{array}{l}\text { first, etanercept } 6 \text { months } \\
\text { second, adalimumab } 4 \text { months }\end{array}$ & infliximab 1 year & etanercept 1 year \\
\hline Onset of TB after anti-TNF therapy & 10 months & 1 year & 1 year \\
\hline
\end{tabular}

\section{References}

1 Global tuberculosis control: WHO Report 2011. http://www.who.int/tb/publications/global_report/en/ chapter 2, p 9.

2 WHO, TB QATAR 2011:

https://extranet.who.int/sree/Reports?op=Replet\&name=/WHO_HQ_Reports/G2/PROD/EXT/TB CountryProfile\&ISO2=QA\&outtype $=$ html.

3 Bassukas ID, Kosmidou M, Gaitanis G, Tsiouri G, Tsianos E: Patients with psoriasis are more likely to be treated for latent tuberculosis infection prior to biologics than patients with inflammatory bowel disease. Acta Derm Venereol 2011;91:444-446.

-4 Silva LC, Silveira GG, Arnone M, Romiti R, Geluk A, Franken KC, Duarte AJ, Takahashi MD, Benard G: Decrease in Mycobacterium tuberculosis specific immune responses in patients with untreated psoriasis living in a tuberculosis endemic area. Arch Dermatol Res 2010;302:255-262.

5 Laffitte E, Janssens JP, Roux-Lombard P, Thielen AM, Barde C, Marazza G, Panizzon RG, Saurat JH: Tuberculosis screening in patients with psoriasis before antitumor necrosis factor therapy: comparison of an interferon-gamma release assay versus tuberculin skin test. Br J Dermatol 2009;161:797-800.

-6 Bordignon V, Bultrini S, Prignano G, Sperduti I, Piperno G, Bonifati C, Filippetti M, Toma L, Latini A, Di Cecio M, Giuliani A, Vocaturo A, Trento E, D’ Agosto G, Francesconi F, Cataldo A, Vento A, Cilenti V, Berardesca E, Ameglio F, Cordiali Fei P, Ensoli F: High prevalence of latent tuberculosis infection in autoimmune disorders such as psoriasis and in chronic respiratory diseases, including lung cancer. J Biol Regul Homeost Agents 2011;25:213-220.

-7 Doherty SD, Van Voorhees A, Lebwohl MG, Korman NJ, Young MS, Hsu S: National Psoriasis Foundation consensus statement on screening for latent tuberculosis infection in patients with psoriasis treated with systemic and biologic agents. J Am Acad Dermatol 2008;59:209-217. 
8 Pathirana D, Ormerod AD, Saiag P, Smith C, Spuls PI, Nast A, Barker J, Bos JD, Burmester GR, Chimenti S, Dubertret L, Eberlein B, Erdmann R, Ferguson J, Girolomoni G, Gisondi P, Giunta A, Griffiths C, Hönigsmann H, Hussain M, Jobling R, Karvonen SL, Kemeny L, Kopp I, Leonardi C, Maccarone M, Menter A, Mrowietz U, Naldi L, Nijsten T, Ortonne JP, Orzechowski HD, Rantanen T, Reich K, Reytan N, Richards $\mathrm{H}$, Thio HB, van de Kerkhof P, Rzany B: European S3-guidelines on the systemic treatment of psoriasis vulgaris. J Eur Acad Dermatol Venereol 2009;23(suppl 2):1-70.

9 Sánchez-Moya AI, Dauden E: Incidence of tuberculosis infection in psoriatic patients on anti-TNF therapy: report of a case series with 144 patients. J Eur Acad Dermatol Venereol 2011;25:730-733.

10 Chiu HY, Hsueh PR, Tsai TF: Clinical experience of QuantiFERON( ${ }^{\circledR}$ )-TB Gold testing in patients with psoriasis treated with tumour necrosis factor blockers in Taiwan. Br J Dermatol 2011;164:553-559.

11 Garcovich S, Ruggeri A, D’Agostino M, Ardito F, De Simone C, Delogu G, Fadda G: Clinical applicability of Quantiferon-TB-Gold testing in psoriasis patients during long-term anti-TNF-alpha treatment: a prospective, observational study. J Eur Acad Dermatol Venereol 2011. DOI: $\underline{10.1111 / j .1468-}$ 3083.2011.04220.x

12 Kardos M, Kimball AB: Time for a change? Updated guidelines using interferon gamma release assays for detection of latent tuberculosis infection in the office setting. J Am Acad Dermatol 2012;66:148-152.

13 Perlmutter A, Mittal A, Menter A: Tuberculosis and tumour necrosis factor-alpha inhibitor therapy: a report of three cases in patients with psoriasis. Comprehensive screening and therapeutic guidelines for clinicians. Br J Dermatol 2009;160:8-15. 